\title{
Investigation of dynamic properties of isotropic and anisotropic magnetorheological elastomers with a hybrid magnet shear test rig
}

\author{
Shaoqi Li, ${ }^{\mathrm{a},}$ Yajun Liang, ${ }^{\mathrm{b}}$ Yancheng Li, ${ }^{\mathrm{a}, \mathrm{c}^{*}}$, Jianchun $\mathrm{Li}^{\mathrm{a}}{ }^{\mathrm{b}}$, and Yadong Zhou ${ }^{\mathrm{b}}$ \\ ${ }^{a}$ School of Civil and Environmental Engineering, University of Technology Sydney, Ultimo 2007, \\ Australia \\ ${ }^{\mathrm{b}}$ School of Civil Engineering, Tianjin Chengjian University, Tianjin 300384, People's Republic of \\ China \\ ${ }^{\mathrm{c}}$ College of Civil Engineering, Nanjing Tech University, Nanjing 211816, People's Republic of China \\ Corresponding author: yancheng.li@uts.edu.au, jianchun.li@uts.edu.au
}

\begin{abstract}
Magnetorheological elastomers (MREs) exhibit instantaneous and reversible adaptability of stiffness and damping properties under the influence of magnetic field, which can be implemented in the development of controllable devices. The main MRE components are normally elastomeric matrix and magnetisable particles. Depending on the distribution of the particles in the matrix, MREs can be classified into isotropic and anisotropic. This work experimentally explored, compared, and modelled the dynamic characteristics of both isotropic and anisotropic MREs with different iron particle weight fractions (17\%, 22\%, and 32\%). A novel shear test rig was designed with hybrid magnets system, i.e., permanent magnet and electromagnets, to fulfil the characterisation tasks. The involvement of the hybrid magnets effectively cuts down the maximum electric current and energy consumption of the rig. The tests were conducted under sinusoidal shear motions with excitation frequency ranging from $0.1 \mathrm{~Hz}$ to $2 \mathrm{~Hz}$ and shear strain varying from $20 \%$ to $60 \%$ to record the force-displacement hysteresis of MRE samples. Four different levels of magnetic field $(0.02,0.54,0.77,1.01 \mathrm{~T})$ were supplied by the hybrid magnetic system were considered in the tests to evaluate the influence of the magnetic fields. Furthermore, characterised hysteretic behaviours for both isotropic and anisotropic MRE were modelled by a strain stiffening phenomenological model with ideal accuracy under the shear excitation inputs and magnetic fields considered.
\end{abstract}

\section{Keywords:}

Anisotropic magnetorheological elastomer; isotropic magnetorheological elastomer; hybrid magnets; MRE shear test; phenomenological modelling 


\section{Introduction}

Magnetorheological elastomers (MREs) exhibit fast and revisable changes of their material properties like stiffness and damping, upon the presence of an external magnetic field. The mechanical properties of these are positively correlated to the strength of the external magnetic field. This unique feature has drawn numerous research and commercial interests; for instance, controllable devices have been developed and investigated to achieve adaptive seismic isolation, and structural control [1-5]. The performance of the aforementioned devices strongly depends on the MRE material adopted. Therefore, understanding the behaviour of the MRE is of significance and should be established prior to the device design in order to obtain a sufficient device performance.

The main MRE components are silicone rubber, silicone oil and ferro-magnetic particles. Up to date, two types of MRE can be characterized which are isotropic and anisotropic MREs, depending on the microstructure formation of the materials. With the presence and absence of a magnetic field during the curing state of MRE fabrication, anisotropic and isotropic MRE can be obtained accordingly. The particles are uniformly distributed in the isotropic MRE. However, the application of the magnetic field during the curing process aligns the ferromagnetic particles thus forming columnar structures embedded in the elastomeric matrix [6]. This structure can be preserved in the MRE after curing thus affecting the mechanical behaviour of the material like stiffness, damping and magnetorheological effect (MR effect) [7]. Not only the structure of the filling particles, but the volume of the iron particle is also an important character that influences the material properties of MRE. Sun et al. measured the relative MR effect of MRE samples with $60,70,80 \%$ weight fractions of Fe content and suggested that the $70 \%$, which is equivalent to approx. $25 \%$ volume fraction, exhibits the highest relative MR effect [8]. In our research, the CIP volume fractions in MREs are extended to 17,22 , and $32 \%$.

The majority of the existing MRE shear test rig and MRE based adaptive device practices solely rely on permanent magnets or electromagnets to generate the magnetic fields, exhibit MR effect, and achieve adjustability of the material properties. Dargahi et al. designed a shear test rig using permanent magnets to obtain the force-displacement relationships and the magnetic flux density was controlled by adjusting the number of permanent magnets (PMs) stacked together or the spacing between the PMs and the MRE samples[9, 10]. Using PMs to adjust the strength of the magnetic field cannot reach the same level of precision and accuracy as using the 
electromagnets. A large portion of shear test rig design features pairs of electromagnetic coils to realise a higher level of accuracy and precision than that of using PM [7, 10-14]. However, this approach still has some limitations. For example, to generate a sufficient amount of magnetic flux, the number of winding and the applied current should be increased to a considerable value which will result in large size of coil and risk of applying high currents. Also, the overheating problem will be raised when the coils are working for a long time. Similarly, these issues are common to happen in the adaptive devices which have a single magnetic source. To mitigate the shortcomings of the devices with a single magnetic field source in the electromagnetic aspect, the concept of using hybrid magnets, i.e., PM and electromagnets have been brought into the development of magnetorheological materials based adaptive devices [15-17].

As an essential step towards the application development, modelling of MRE are frequently investigated. The phenomenological model acts a major role to predict the force-displacement relationships of the materials, due to its versatile and simple formulation process. Li et al. developed a four-parameter model that accurately depicts the viscoelastic properties of MRE [18]. Addressing the magnetic field dependency of MRE, a generalised Prandtl-Ishlinskii model was constructed by Dargahi et al. to capture the magnetic-field-induced unique hysteresis behaviour for isotropic MREs [9]. Reflecting on the strain stiffening effect generated by MRE, Yu et al. proposed a phenomenological model which shows ideal fit with the experimentally characterised hysteresis behaviours of an MRE based isolator [19].

In this research, a novel and compact material test rig featuring both permanent magnet (PM) and electromagnets was designed for MRE shear tests. Finite element modelling was conducted using ANSYS Electronics Desktop to reveal the electromagnetic performance of the shear test rig. Isotropic and anisotropic MREs with three different CIP volume percentages $(17 \%, 22 \%$, and $32 \%$ ) were tested by the proposed test rig. The discussions on the influences of CIP volume fraction and microstructure of MRE on the material performance were carried out. Furthermore, phenomenological modelling was conducted to predict the force-displacement relationships of both isotropic and anisotropic samples.

\section{Shear test rig featuring both PM and electromagnets}

\subsection{Design of the MRE shear test rig}

Figure 1 (a) shows the schematic diagram of the proposed shear test rig. It includes a hybrid magnets system which features two electromagnetic coils and a PM. The size of the test rig is 
$200 \mathrm{~mm} \times 180 \mathrm{~mm} \times 85 \mathrm{~mm}$. The dimension of PM and steel blocks is $35 \mathrm{~mm} \times 35 \mathrm{~mm} \times 5$ $\mathrm{mm}$. As shown in figure 1 (b), the shear plate contains a $45 \mathrm{~mm} \times 70 \mathrm{~mm} \times 15 \mathrm{~mm}$ copper block plate, two steel blocks and one PM. The copper block was extruded cut by a $35 \mathrm{~mm} \times 35$ $\mathrm{mm}$ square through the thickness direction to accommodate the stacked steel blocks and PM. Two fabricated MRE samples and the shear plate were sandwiched between the left and right steel fixtures to form a double-lap structure. The MRE samples were centred on the steel blocks and the fixtures. The size of the fixture is $62.5 \mathrm{~mm} \times 35 \mathrm{~mm} \times 35 \mathrm{~mm}$. The two fixtures were bolted to the top of a c-shaped steel core to form an enclosed magnetic flux path. The two coils were installed on each branch of the steel core. Each coil contains 1900 turns of coated wire. The winding directions of the coils are opposite to each other to generate magnetic fluxes of the same clockwise or anticlockwise direction. N40 grade NdFeB PM was chosen for this test rig. Two load washers were installed at one end of the fixture to gauge the applied compression force to the MRE samples before the left and right fixtures were bolted tight to the steel core.

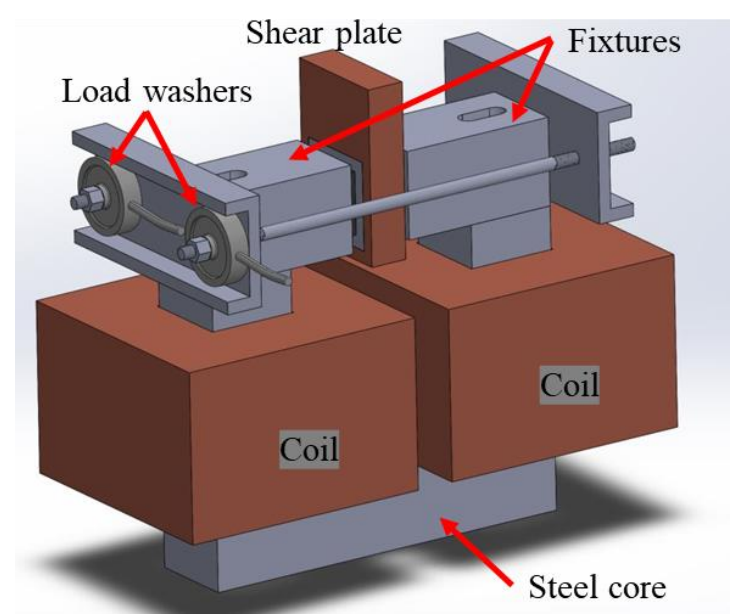

(a) The proposed shear test rig

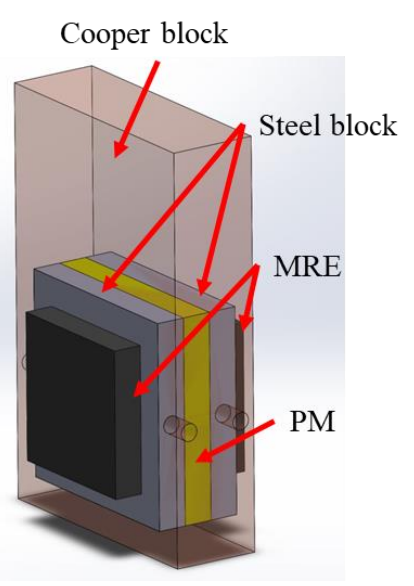

(b) The shear plate and MRE samples

Figure 1. The schematic diagram of the proposed MRE shear test rig

\subsection{Finite element modelling}

Finite element analysis (FEA) was conducted using ANSYS Electronics Desktop to characterize the magnetic field distribution in MRE samples under scenarios with different currents applied to the coils. The model was constructed following the designed test rig dimensions and materials. The N40 NdFeB PM was modelled with remanence $B_{\text {rem }}=1.25 \mathrm{~T}$ and coercivity $H_{\mathrm{c}}=9.5 \times 10^{5} \mathrm{Am}^{-1}$. The nonlinear B-H curves of MRE and steel used in this finite element model are shown in figure 2. It should be noted that the B-H curve for MRE used in this model is obtained from MRF-132DG magnetorheological fluid by Lord Corporation due to similar weight fraction of iron particle content. 

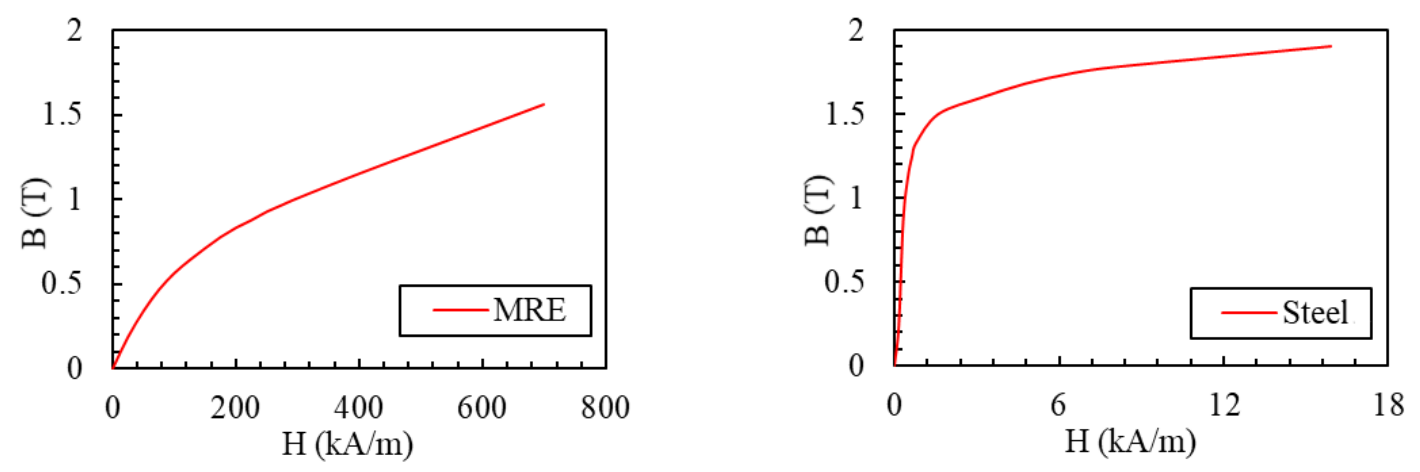

Figure 2. B-H curves of MRE and steel

Figure 3 presents the FEA results for scenarios where the applied currents vary from $0.9 \mathrm{~A}$, $0.66 \mathrm{~A}, 0.43 \mathrm{~A}, 0.2 \mathrm{~A}, 0 \mathrm{~A}$ and $-0.45 \mathrm{~A}$. It should be noted that upon the application of positive value current, the coils produce magnetic fluxes which travel opposing to the direction of the flux generated by the PM. Thus, the positive currents are meant to reduce the B values in the MRE. As observed in figure 3 (a), the flux density is almost zero $(0.02 T)$ at the positions of the MRE samples. This indicates that the coils are capable of cancel out the 'bias' field provided by the PM with $0.9 \mathrm{~A}$ applied. By cutting down the applied to $0.43 \mathrm{~A}$ and $0 \mathrm{~A}$, as shown in figure 3 (b) and 3 (c), the flux density in MRE samples rises to $0.54 \mathrm{~T}$ and $0.77 \mathrm{~T}$, respectively.

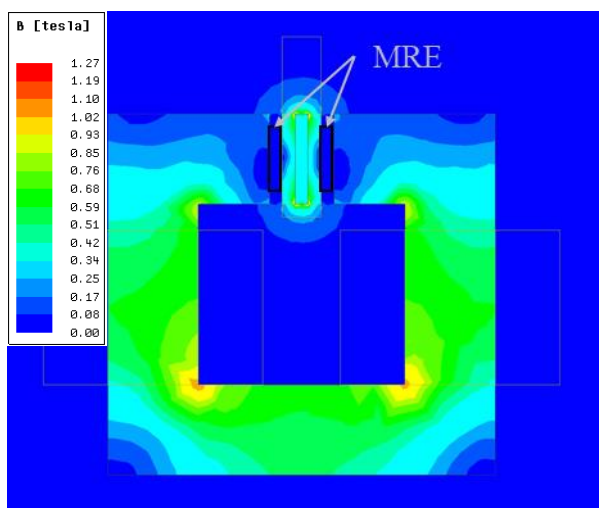

(a) 0.9 A applied

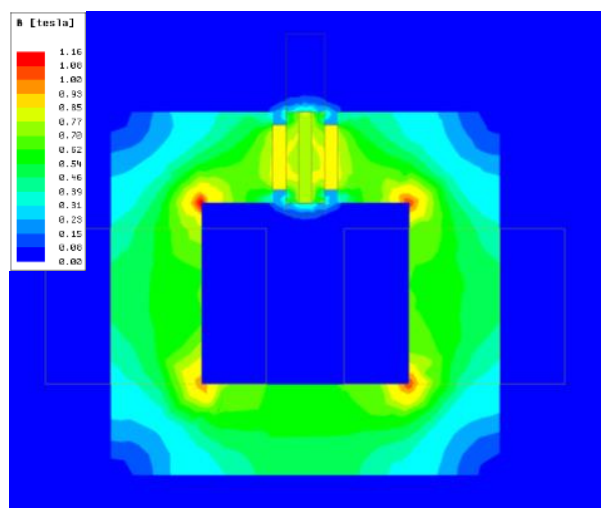

(c) 0 A applied

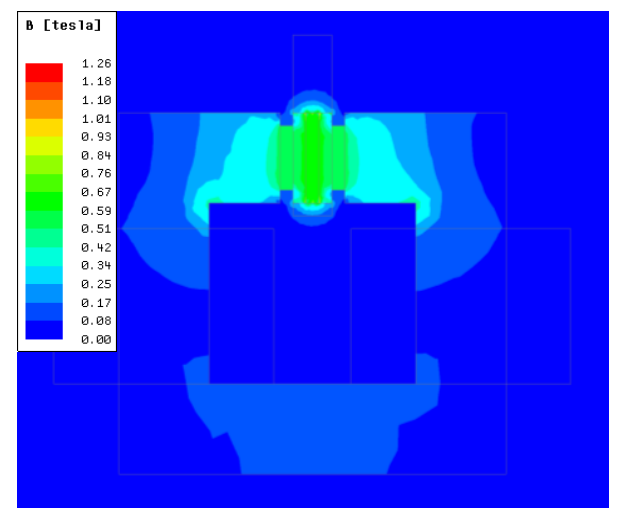

(b) 0.43 A applied

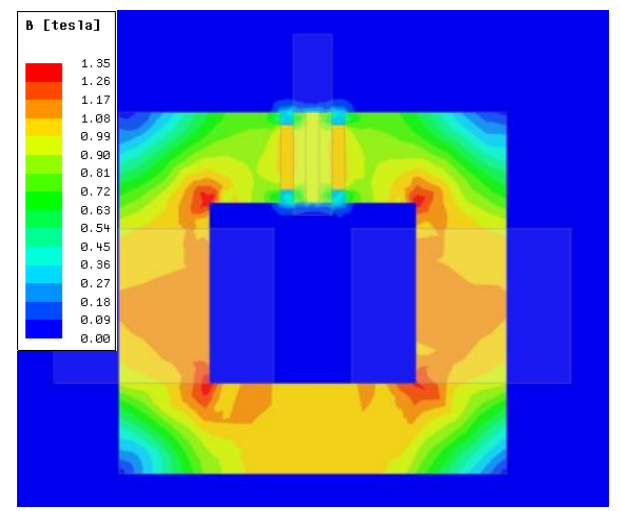

(d) -0.45 A applied

Figure 3. FEA results 
The relationship between flux density values in the MRE sample and the applied current to each coil are summarized in figure 4.

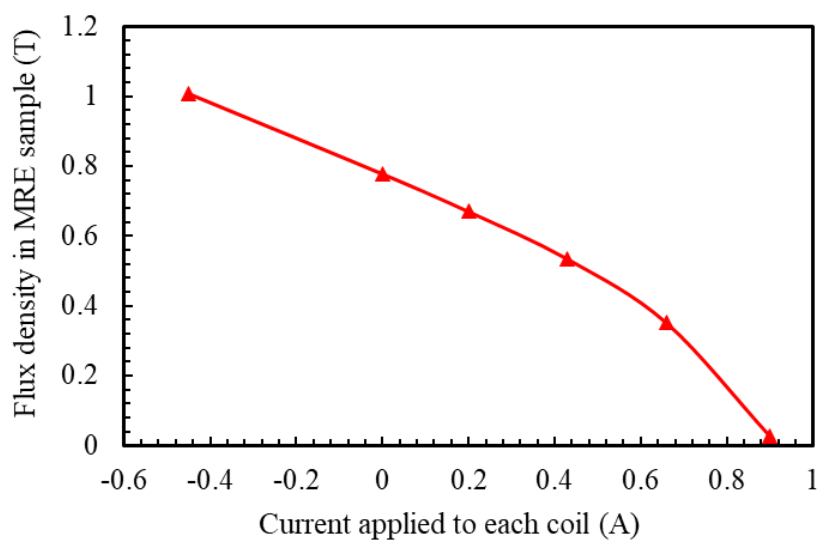

Figure 4. Flux densities in MRE sample

The flux density in the MRE sample decreased to $0.02 \mathrm{~T}$ from 1.01T when the applied current increased from -0.45 A to 0.9 A. At 1.01T, the influence of the magnetic field to the adjustability of MRE reaches saturation and this will be further validated by experiments in the following section.

\section{Experiments}

\subsection{Material preparation}

The materials required to produce the MREs in this research are silicone elastomer base (SYLGARD 184, Dow Inc., United States), silicone oil (Type H201-500, Shanghai Hushi Co., Ltd., China) and 3-5 $\mu$ m diameter CIP (Beijing Xing Rong Yuan Technology Co., Ltd., China). To fabricate isotropic MREs, the first step is to mix and stir the silicone rubber and the silicone oil and CIP at $1000 \mathrm{rpm}$ for 15 minutes. The weight ratio between the silicone rubber and silicone oil is $1: 1$. Then, the mixture was treated with an ultrasonic vibrator for 15 minutes to obtain a more uniform distribution of CIP and remove the air bubble in the mixture. To further eliminate the air bubble, the mixture was stored in the vacuum drying machine for 10 minutes. After this process, the mixture was cast into the mould and cured in the oven at $80{ }^{\circ} \mathrm{C}$ for 2 hours. Isotropic MREs can be obtained after curing. As for the preparation of anisotropic MREs, the difference lies in the curing stage. $0.5 \mathrm{~T}$ magnetic field was generated by coils and applied to the mixture in the mould and the curing temperature was maintained at $80{ }^{\circ} \mathrm{C}$ for 2 hours by the heater. The direction of the magnetic field is parallel with the thickness direction of the 
samples. Thus, the formed chain-like structure also aligns with the thickness direction after curing. It should be noted that the material of the mould and the heater must be non-magnetic materials to ensure the uniformity of the magnetic field. A total of six types of MRE samples were prepared and the details of the samples are summarised in table 1.

Table 1. Details of MRE samples

\begin{tabular}{c|c|c|c|c|c}
\hline Material ID & MRE type & CIP vol\% & Width & Height & Thickness \\
\hline iso17 & isotropic & $17 \%$ & & & \\
aniso17 & anisotropic & $17 \%$ & & & \\
iso22 & isotropic & $22 \%$ & & & \\
aniso22 & anisotropic & $22 \%$ & $25 \mathrm{~mm}$ & $25 \mathrm{~mm}$ & 5 \\
iso32 & isotropic & $32 \%$ & & & \\
ansio32 & anisotropic & $32 \%$ & & & \\
\hline
\end{tabular}

\subsection{Observation of microstructure}

The microstructures of all six MRE samples were viewed by scanning electron microscope (SEM) machine from Hitachi, Ltd. (model TM4000) at 500 times magnification with $15 \mathrm{kV}$ accelerating voltage. Since the chain-like structures in the anisotropic samples are parallel with the thickness direction, the anisotropic samples were cut in the thickness direction prior to SEM observation. Figure 5 shows the SEM micrographs of MRE samples, where figure 5 (a), (c) and (e) represents the results for the isotropic samples with 17\%, 22\% and 32\% CIP volume fractions, respectively, and figure 5 (b), (d) and (f) are for the micrographs of anisotropic samples with $17 \%, 22 \%$, and $32 \%$ CIP volume fractions, correspondingly. It should be noted that the difference in the directions of the chain structure is due to the placing of the sample during SEM observation. For all anisotropic samples, the chains are parallel to the thickness direction of the sample. By comparing the results between isotropic and anisotropic MRE samples, chain-like structures can be clearly observed in anisotropic samples. As the existence of the chain-like structures, the distances between CIPs is closer along the thickness direction for the anisotropic MREs than that of isotropic materials. 


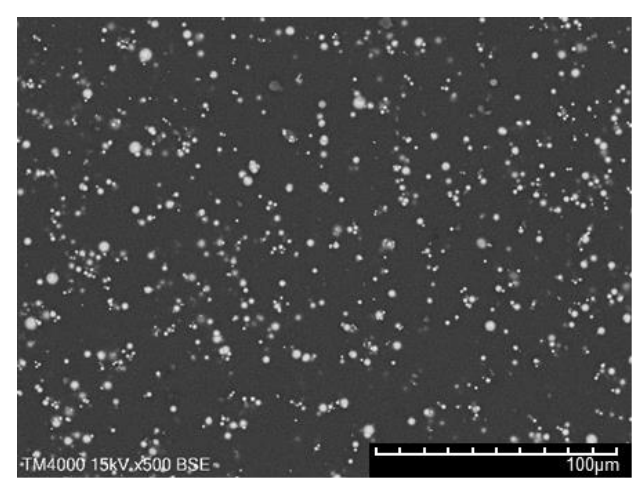

(a) iso17

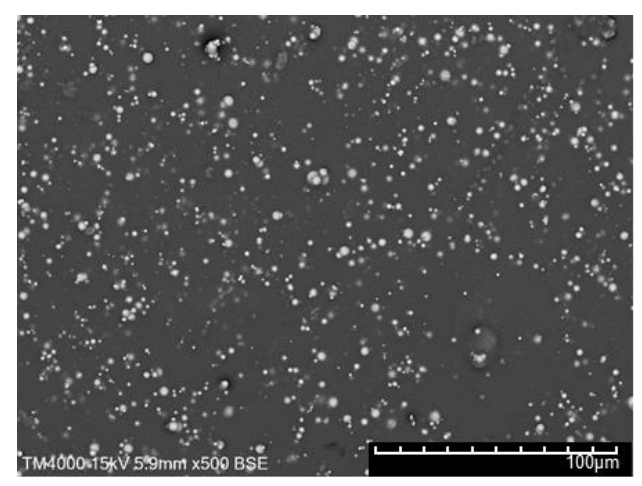

(c) iso22

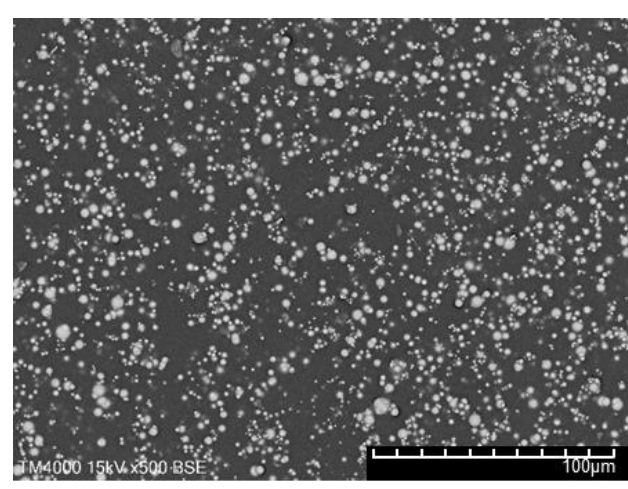

(e) iso32

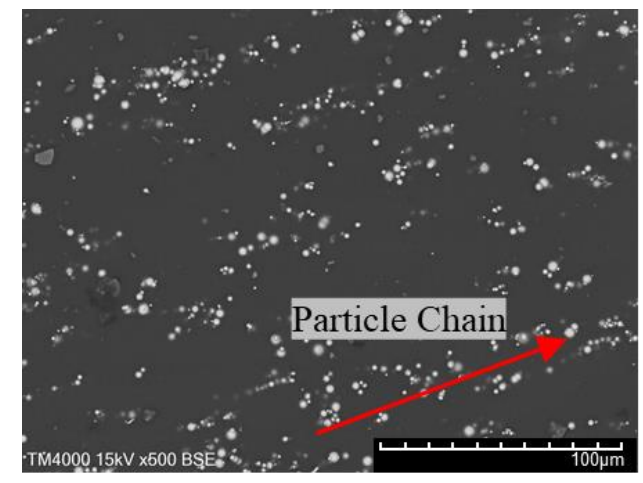

(b) ansio17

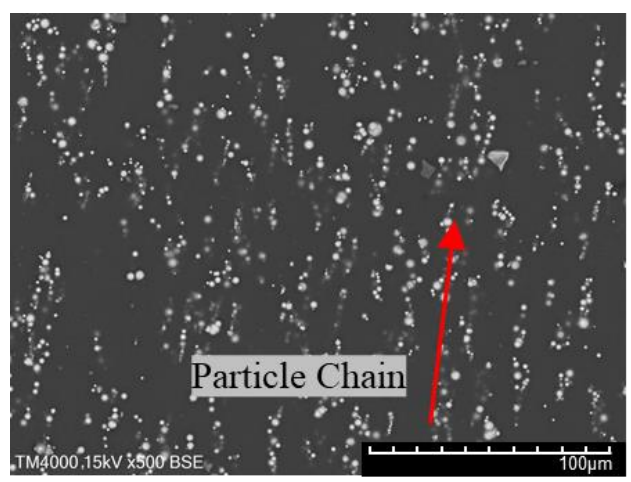

(d) aniso22

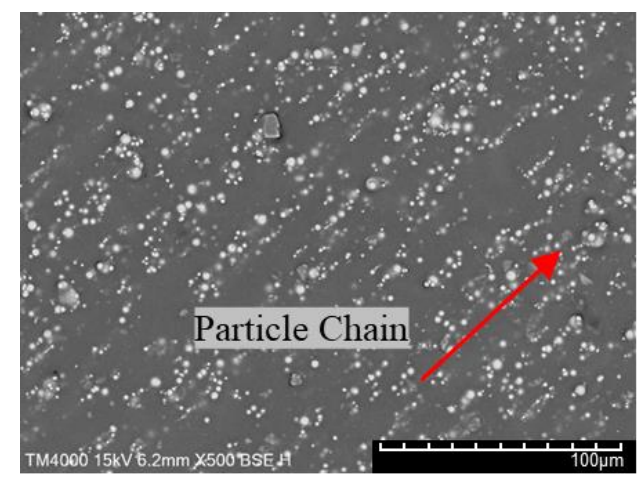

(f) $\operatorname{aniso32}$

Figure 5. Micrographs of isotropic and anisotropic MRE samples (red arrows indicate the direction of the particle chains)

\subsection{Test set up}

Experiments were conducted to obtain the dynamic behaviours of all six types of MRE samples under various shear amplitude, excitation frequency and applied current. The test set up for the MRE shear test is presented in figure 6. A universal testing machine (model: WAW-B, Jinan Shenggong Testing Machine Co., Ltd, China) was used for the experiments in displacement control mode. A load cell is adapted to the top of the shear plate. The other end of the load cell is connected to an adaptor which is gripped by the bottom crosshead. A linear variable displacement transducer (LVDT) was attached to the top crosshead to measure the 
displacement of the bottom crosshead. The two coils are connected to separate direct current power suppliers but are applied with identical current during the test.

The force-displacement hysteresis loops of the MRE samples were recorded by using sinusoidal excitations with $0.1 \mathrm{~Hz}, 0.5 \mathrm{~Hz}, 1 \mathrm{~Hz}$ and $2 \mathrm{~Hz}$ set as excitation frequencies and $1 \mathrm{~mm}$, $2 \mathrm{~mm}$ and $3 \mathrm{~mm}$ chosen as amplitude. Considering the thickness of each MRE sample is $5 \mathrm{~mm}$, $1 \mathrm{~mm}, 2 \mathrm{~mm}$ and $3 \mathrm{~mm}$ amplitudes are corresponding to $20 \%, 40 \%$ and $60 \%$ shear strain. The applied currents to the coils are $0.9 \mathrm{~A}, 0.66 \mathrm{~A}, 0.43 \mathrm{~A}, 0.2 \mathrm{~A}, 0 \mathrm{~A}$ and $-0.45 \mathrm{~A}$, respectively. During each test, the universal testing machine produces an excitation signal at a fixed frequency and amplitude and the applied current was held at a constant level. Each test was repeated four times to ensure the reliability of the test results. Therefore, a total of 72 hysteresis loops were obtained for each type of material under the designed loading conditions and applied currents. The sampling rate of the data acquisition device was set at $500 \mathrm{~Hz}$.
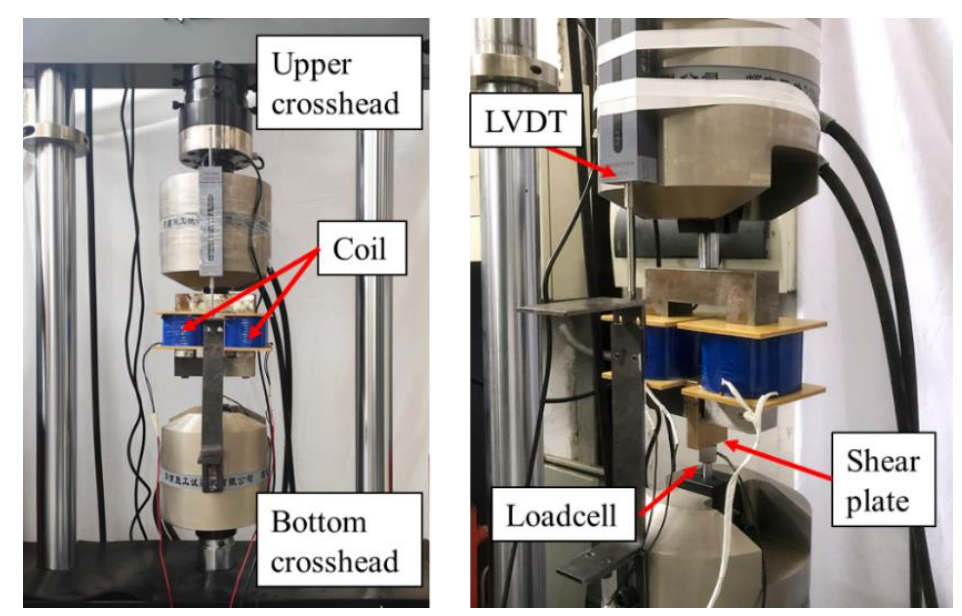

Figure 6. Experimental set up

\section{Experimental results and discussion}

\subsection{MRE hysteresis characteristics}

The measured force-displacement hysteresis loops indicated that the dynamic behaviour of the MRE samples are strongly dependent on the excitation amplitude and magnetic field applied to the MRE samples. Figure 7 comparatively presented the effects of excitation frequency, amplitude and applied current for both isotropic and anisotropic MRE samples. Due to the large amount of data, the measured hysteretic results for samples with the $17 \%$ CIP volume fraction were presented as examples. It should be noted that the forces in figure 7 are half of the measured forces, due to the double-lap configuration of the test rig. 


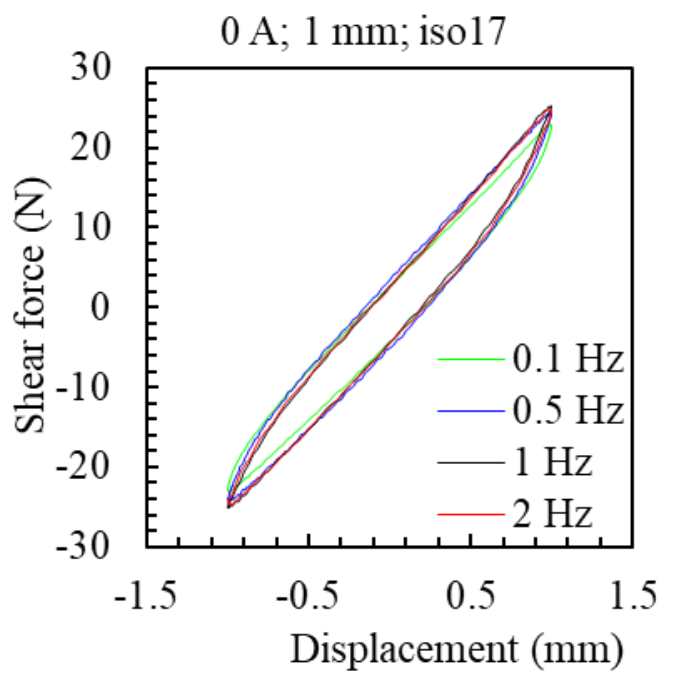

(a) Effect of frequency (iso17)

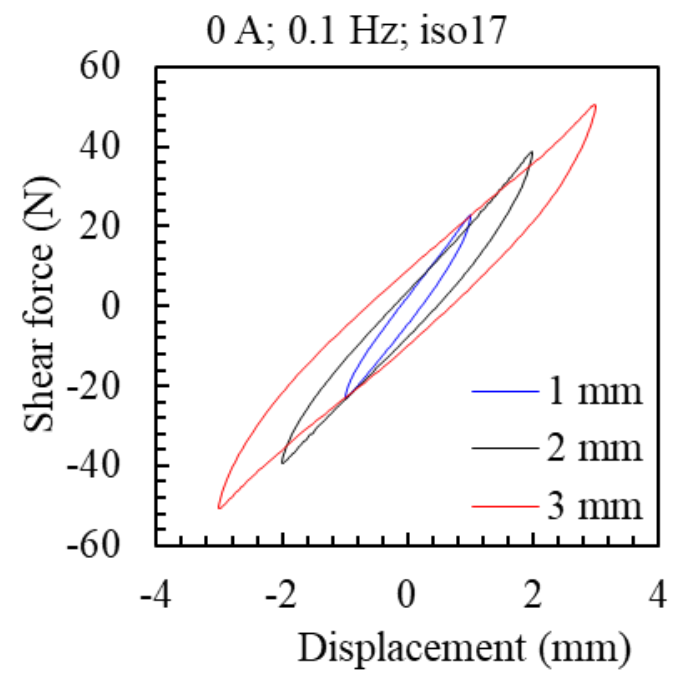

(c) Effect of amplitude (iso17)

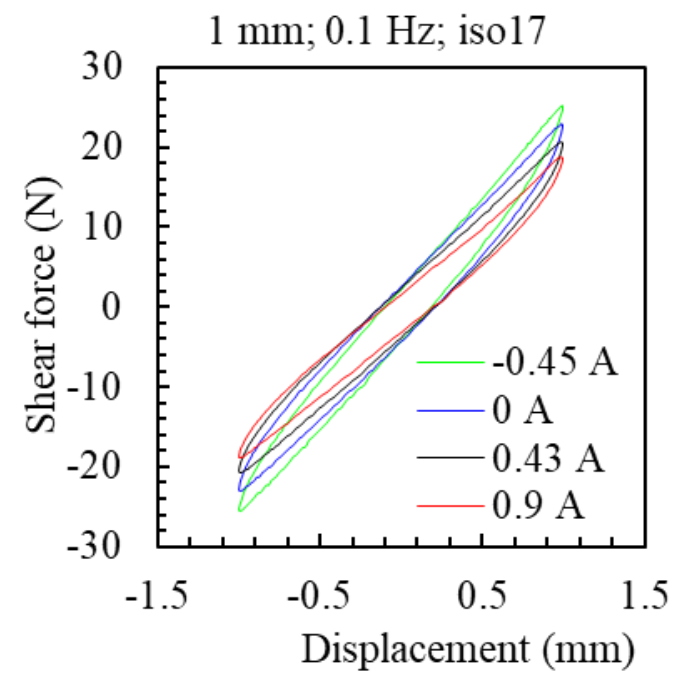

(e) Effect of current (iso17)

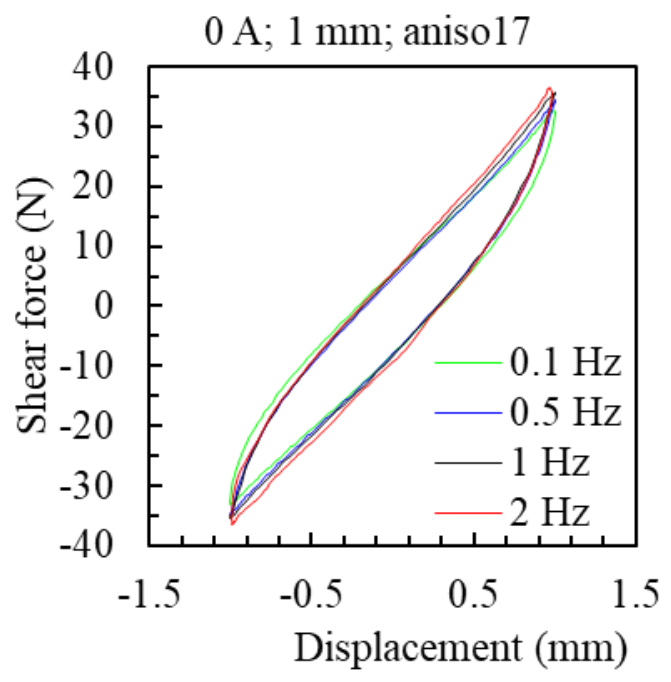

(b) Effect of frequency (aniso17)

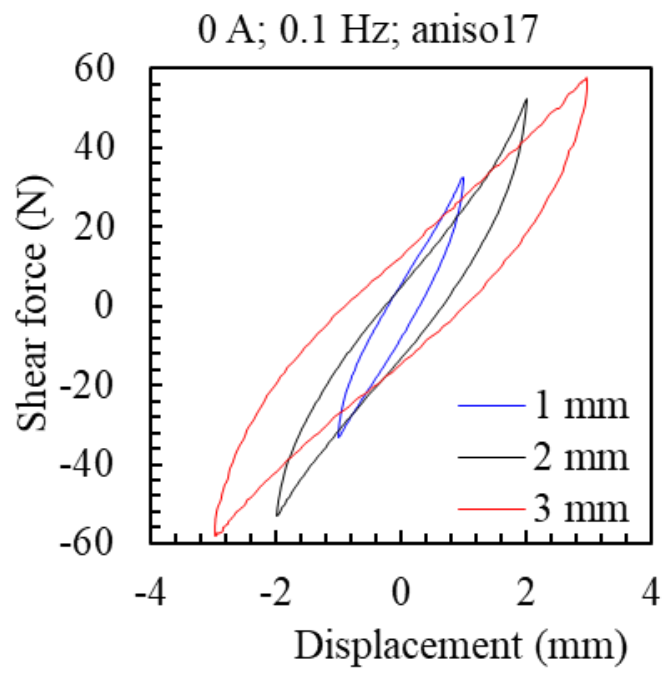

(d) Effect of amplitude (aniso17)

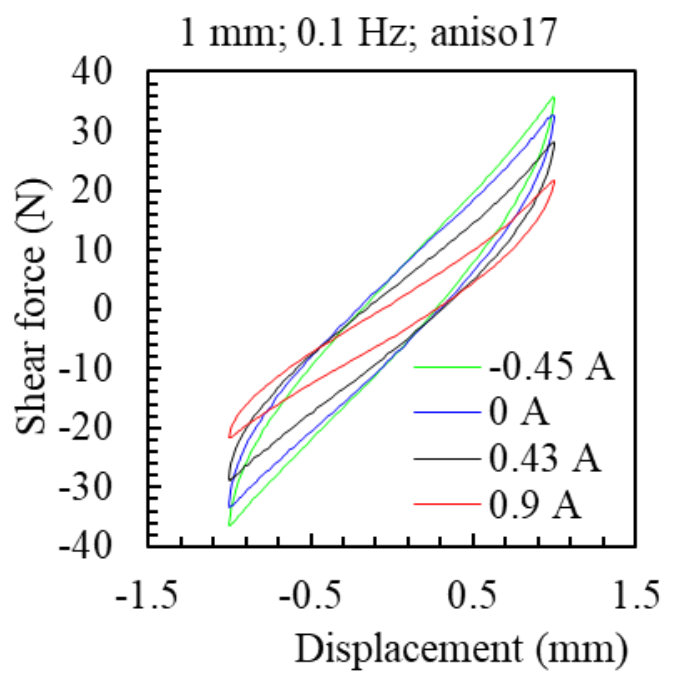

(f) Effect of current (aniso17)

Figure 7. Recorded shear force-displacement hysteresis loops 
From figure 7 (a) and (b), which are responses of the isotropic and anisotropic MRE under different excitation frequency, slight increases of maximum forces and dissipated energy (enclosed area of hysteresis loop) can be observed. This phenomenon is identical to the findings from the investigations conducted by [9]. Comparing the discrepancies of the performances between isotropic and anisotropic samples, under the same loading condition, the enclosed areas of the anisotropic sample are larger than that of the isotropic sample. This suggests the better energy dissipating property of anisotropic MRE.

In figure 7 (c) and (d), when running the shear excitations with different amplitudes, the areas of the dissipated and the maximum forces grow significantly with the increase of excitation amplitude. When adding of excitation amplitude, the maximum forces of isotropic sample grow steadily; whereas, the anisotropic has a much larger force increase when the amplitude grow from $1 \mathrm{~mm}$ to $2 \mathrm{~mm}$ than that of the increment from $2 \mathrm{~mm}$ to $3 \mathrm{~mm}$. This indicates more linear mechanical properties of isotropic samples under the change of excitation amplitude when compared with anisotropic samples.

In figure 7 (e) and (f), the increase of applied currents to the coils leads to reductions in dissipated energy and maximum force for both isotropic and anisotropic samples effectively. The effect of applied magnetic field on the anisotropic samples is greater than on the isotropic samples. This suggests the higher adjustability in the material performances of anisotropic samples. Owing to the pre-aligned CIP chain along the direction of magnetic fluxes in anisotropic samples, the distances between CIPs are shorter in the direction of fluxes than that in the anisotropic samples thus resulting in this significant improvement of MR effect for anisotropic samples [7].

The excitation frequency, amplitude and applied current have similar influences on the forcedisplacement hysteretic performance for both isotropic and anisotropic materials. Nevertheless, the anisotropic samples exhibit higher forces than that of isotropic samples under the same excitation. With varying applied current, anisotropic samples have a larger range of adjustability. Differences in the shape of the hysteresis loops for the anisotropic and isotropic samples should also be noted. The slopes at the tips of hysteresis loops show an increasing trend for anisotropic samples, and, this phenomenon is less obvious in the hysteresis loops of isotropic materials due to presence of pre-aligned CIP chain structure in anisotropic MREs.

\subsection{Effective stiffness}

To further evaluate the influences of excitation inputs and magnetic fields on the material properties of all six types of MRE samples, effective stiffness values were calculated from the hysteresis loops. Effective stiffness can be represented by Eq.1 [1]: 


$$
K_{\mathrm{eff}}=\frac{F_{\mathrm{dmax}}-F_{\mathrm{dmin}}}{\Delta_{\max }-\Delta_{\min }}
$$

Where $F_{\mathrm{dmax}}$ and $F_{\mathrm{dmax}}$ are the forces measured at the maximum displacement $\left(\Delta_{\max }\right)$ and minimum displacement $\left(\Delta_{\max }\right)$ in one loading cycle, respectively. Figure 8 illustrates the effects of applied current, excitation frequency and amplitude on the effective stiffness of MRE samples. As observed in figure 8, anisotropic samples exhibit higher effective stiffness compared to the isotropic samples with the same CIP content under the same test condition. This trend matches well with the finding from the investigation of rheological properties of isotropic and anisotropic MRE conducted by $\mathrm{Lu}$ et al. [7]. For samples with the same microstructure, higher CIP content results in higher effective stiffness. From figure 8 (a), with the applied field increasing from $0.02 \mathrm{~T}$ to $1.01 \mathrm{~T}$, the effective stiffness of all MRE samples increase to the highest at $1.01 \mathrm{~T}$. Anisotropic samples exhibited a larger range of stiffness change than isotropic samples under the influence of applied current. However, in the scenario where the maximum current applied, the effective stiffness of ansio32 sample dropped to a lower value than that of the iso32 sample. For $17 \%$ and $22 \%$ CIP contents samples, the differences of effective stiffness between anisotropic samples and isotropic samples became smaller with the increase of applied current. Figure 8 (b) and (c) indicate that the effective stiffness of all MRE samples is positively correlated to the excitation frequency and negatively corrected to the shear amplitude.

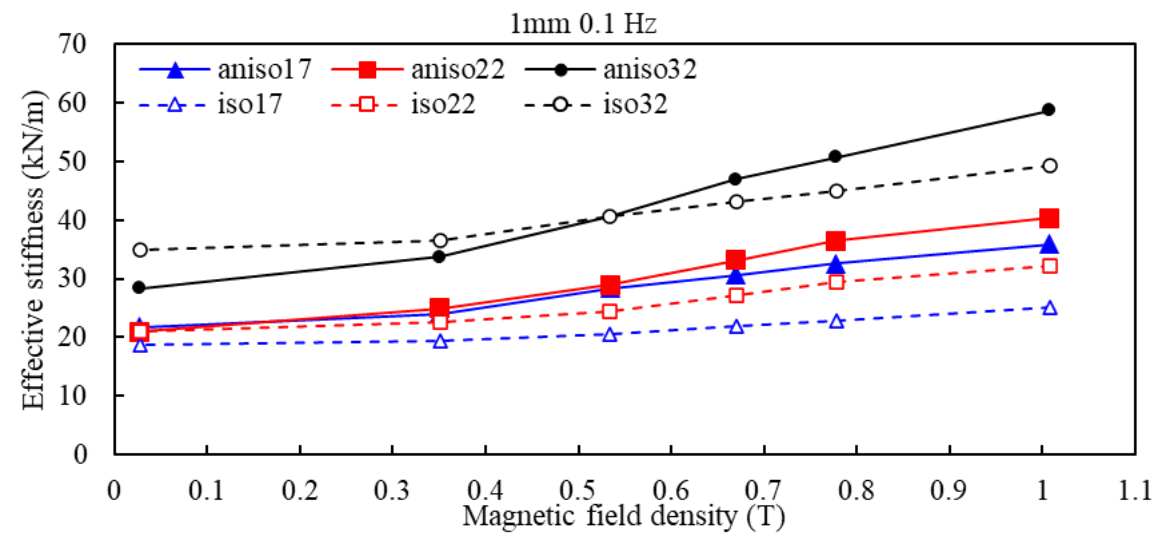

(a) Effects of the external magnetic field 


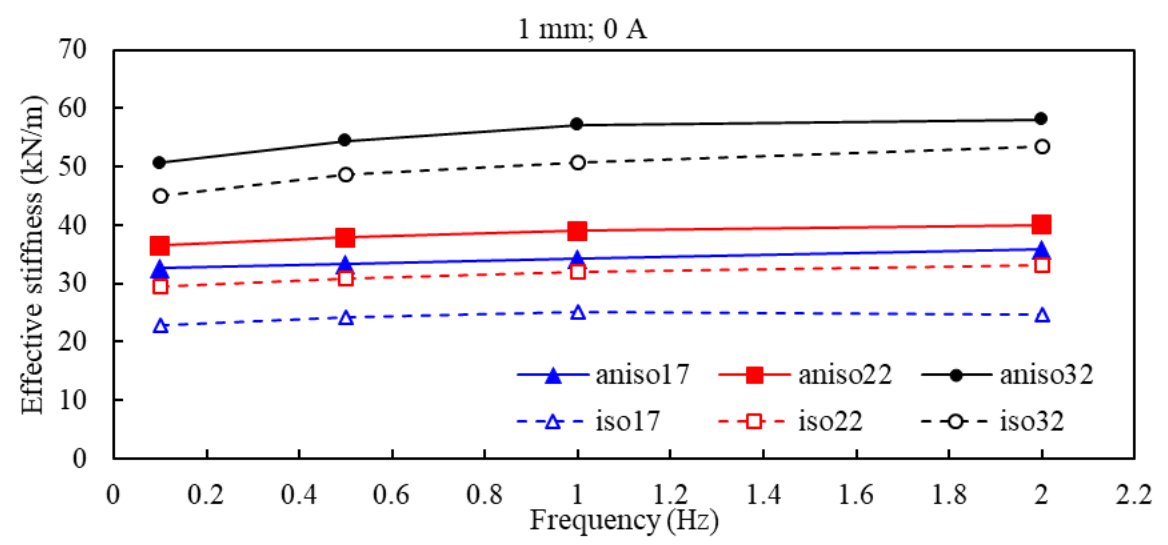

(b) Effects of frequency

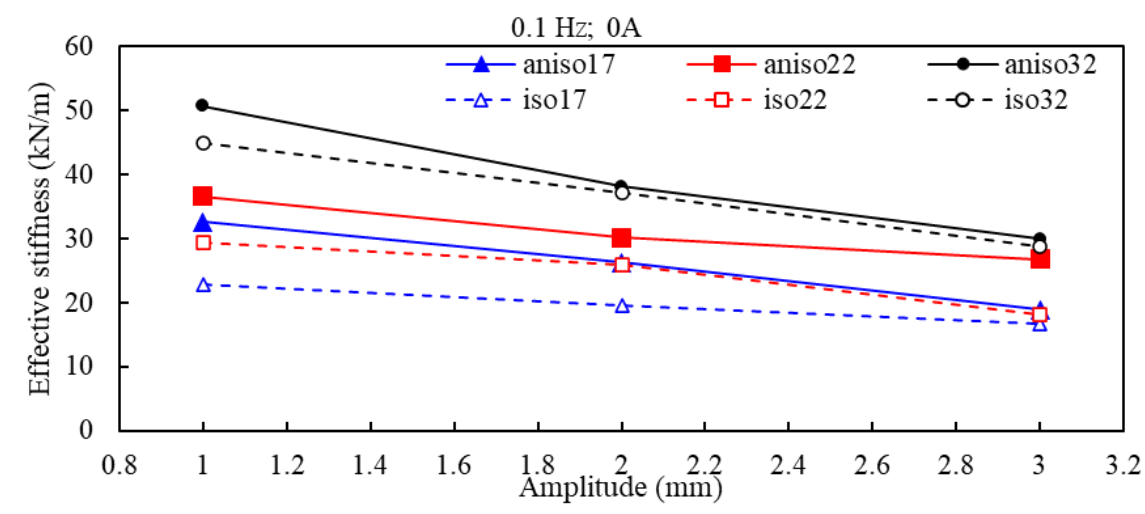

(c) Effects of amplitude

Figure 8. The effective stiffness of MRE samples under influences of magnetic field, frequency, and amplitude

Figure 9 marks the percentage of increase of effective stiffness between the minimum (-0.45 A) and maximum ( $0.9 \mathrm{~A})$ currents applied scenarios to quantitively analyse the adjustability of all six MRE samples under various excitation inputs. In figure 9 (a), which summarises the 0.1 $\mathrm{Hz}$ excitation scenario, anisotropic samples exhibit wider adaptive ranges that that of isotropic samples. Ansio32 has the highest increase\% which is $106.61 \%$ under $1 \mathrm{~mm}$ and $0.1 \mathrm{~Hz}$ excitation; however, this value decreases to $25.90 \%$ as the amplitude increased to $3 \mathrm{~mm}$. Though anisotropic samples have higher adjustability, more stable adaptive ranges can be observed in isotropic samples throughout the $1 \mathrm{~mm}$ to $3 \mathrm{~mm}$ amplitude range. This phenomenon could be explained by the difference between the microstructures in MRE samples. In anisotropic samples, ferro-magnetic particles are closely lined up as columnar structures along the travelling direction of fluxes, as shown in figure 5 (b), (d), and (f). When the columnar structures are subjected to the shear deformation perpendicularly, they become stretched; and, the distribution of CIP deviates significantly. As for isotropic sample, the filling particles are uniformly distributed in the elastomeric matrix. Since the magnetic forces between ferro-magnetic particles are highly dependent on the distance between the particles, the 
adjustability of the anisotropic samples become more dependent on the excitation amplitude than the isotropic samples. To present the influence of excitation frequency on the adjustability, figure 9 (b) summarises the increase\% against excitation frequency for the $1 \mathrm{~mm}$ amplitude scenario. Negligible influences of excitation frequency to the adaptabilities of all six types of MRE samples can be observed.

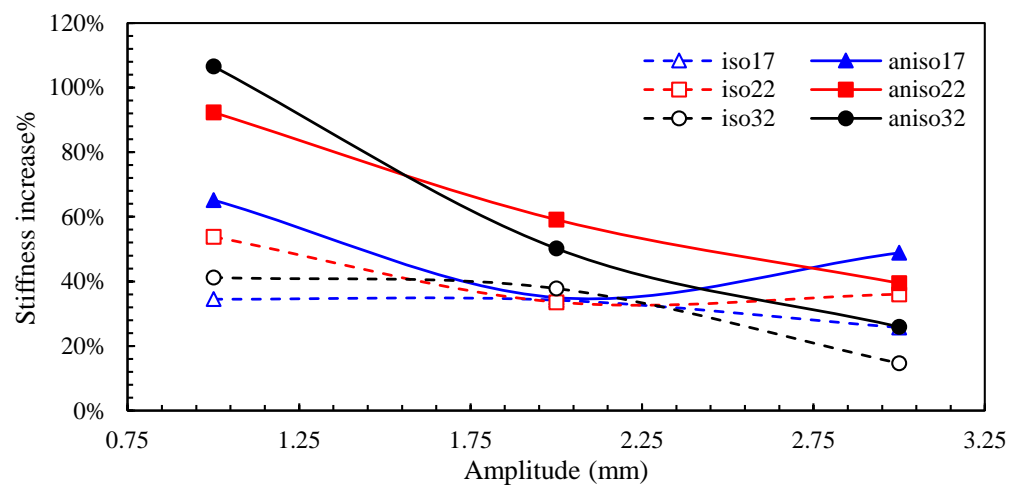

(a) Influence of amplitude under $1 \mathrm{~Hz}$ frequency

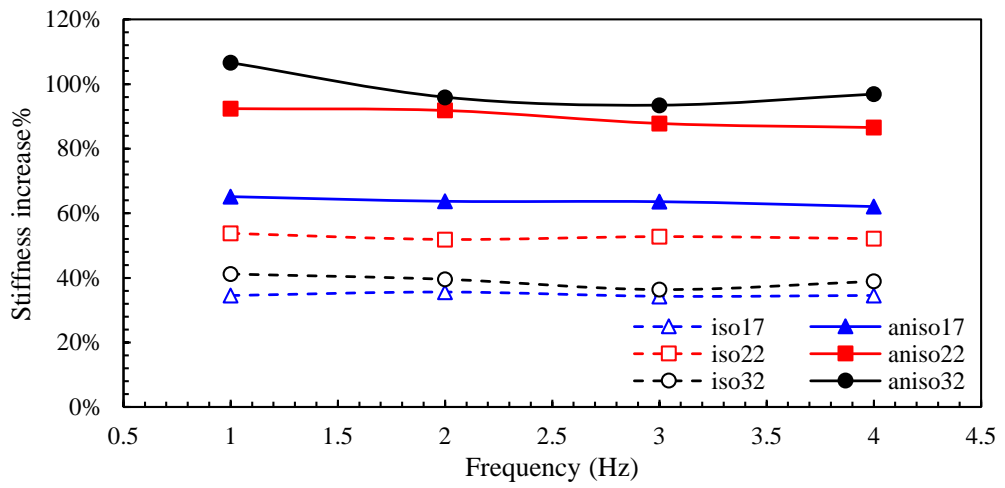

(b) Influence of frequency under $1 \mathrm{~mm}$ amplitude

Figure 9. Increase percentage of effective stiffness of MRE samples

\subsection{Equivalent damping}

Though the enclosed area of hysteresis loop indicates the energy dissipated during shear motion, equivalent damping was calculated to precisely characterize the performance of MRE samples in dissipating energy, following Eq.2 [1]:

$$
C_{e q}=\frac{E}{2 \pi^{2} f A^{2}}
$$

where $\mathrm{E}$ is the enclosed area of the corresponding hysteresis loop, $f$ is the excitation frequency and $A$ is the excitation amplitude. 
Figure 10(a), (b) and (c) show the variances of equivalent damping for six MRE samples subjected to the influences of the applied field, excitation frequency and amplitude, respectively. The damping performances behave similarly to the effective stiffness which increases with the increases of the applied field; however, decreases with the increase of excitation amplitude and frequency. Excitation frequency acts a significant role in the damping performance of MRE samples. A sharp decreasing trend can be observed when the excitation frequency increases from $0.1 \mathrm{~Hz}$ to $2 \mathrm{~Hz}$. It is worth noting that, at lower loading frequencies $(0.1$ and $0.5 \mathrm{~Hz})$, the discrepancies of damping performances between different samples are apparent. Anisotropic samples have higher equivalent damping values than isotropic samples. Also, higher CIP percentage results in higher equivalent damping.

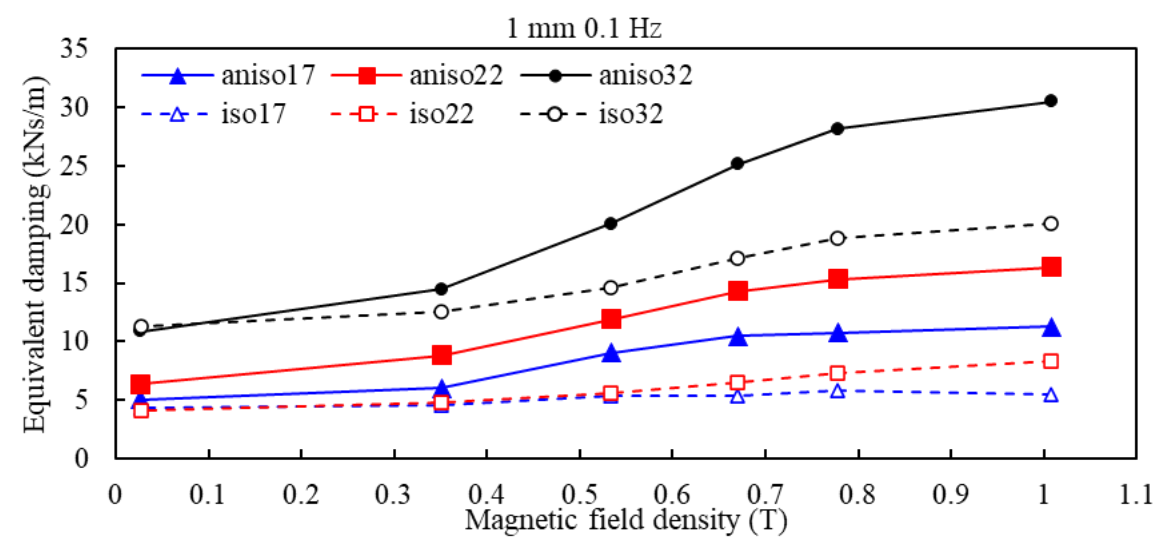

(a) Effects of the external magnetic field

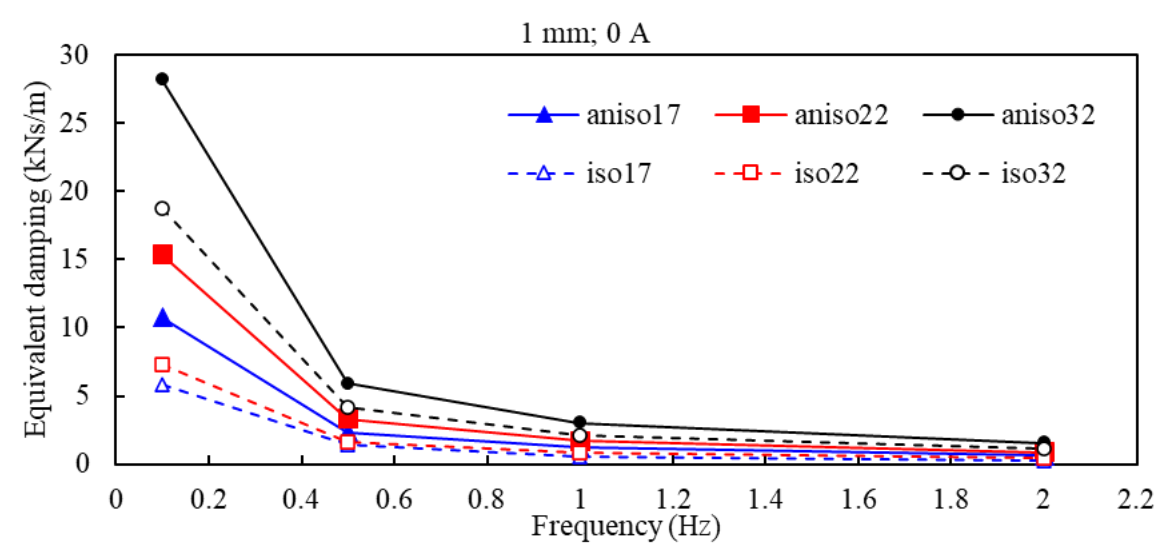

(b) Effects of frequency 


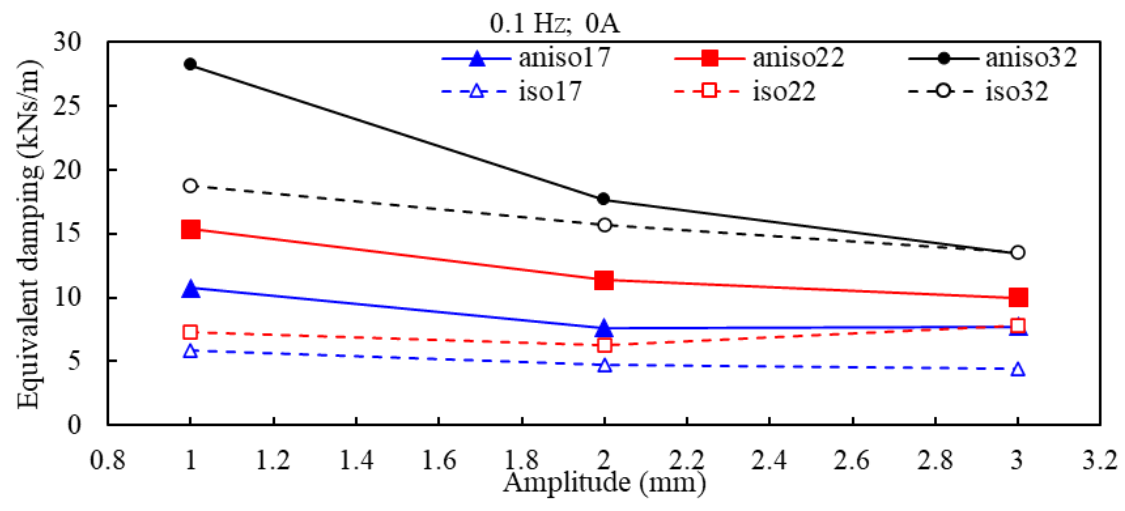

(c) Effects of amplitude

Figure 10. Equivalent damping of MRE samples under influences of magnetic field, frequency, and amplitude

\section{Modelling of isotropic and anisotropic MRE}

The results of the MRE shear test indicate that viscoelastic and strain stiffening are the two major features of both isotropic and anisotropic samples. With the intensifying of the magnetic field, the force-displacement hysteresis reflects a stronger stain stiffening effect. Hence, a simplification from the strain stiffening model proposed by $\mathrm{Yu}$ et al. was adopted in the modelling of the hysteretic responses for iso17 and aniso17 MRE samples, as shown in figure 11 [19]. The model contains three parts which are an elastic spring element $(k)$, damper viscous damping element $(c)$ and the strain stiffening component. The $F_{0}$ term is not included in this model since the experiment was conducted after the initial shear force was eliminated by adjusting the crosshead of the material testing machine. The model is formulated as follow:

$$
F=k x+c \dot{x}+a|x| x^{3}
$$

where $\mathrm{F}$ is the estimated shear force, $x$ and $\dot{x}$ represent the displacement and velocity of the shear movement, and $k, c$, and $a$ are the parameters for the linear spring, dashpot, and stress stiffening element, respectively. 


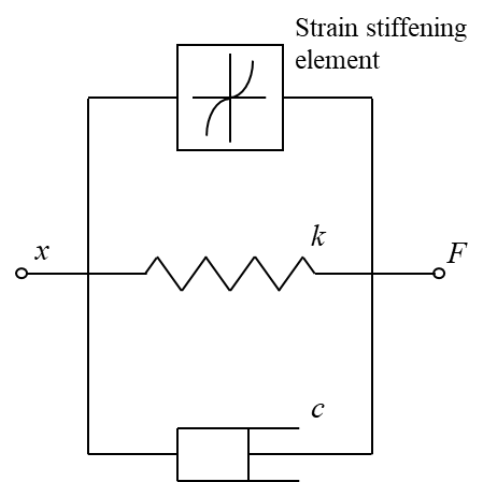

Figure 11. The strain stiffening phenomenological model [19]

The combination of the model parameters, $k, c$, and $a$, that yields the minimum error between the estimated shear force and experimental data was solved by the least square method. Figure 12 and 13 present the modelling results under the varying of excitation frequencies, amplitudes, and magnetic fields for iso17 and ansio17 MRE samples, respectively. The modelling results suggest that the unique stain stiffening effect and field-dependent viscoelastic properties of both isotropic and anisotropic samples can be well predicted by the stain stiffening phenomenological model under the excitation inputs and magnetic field considered.

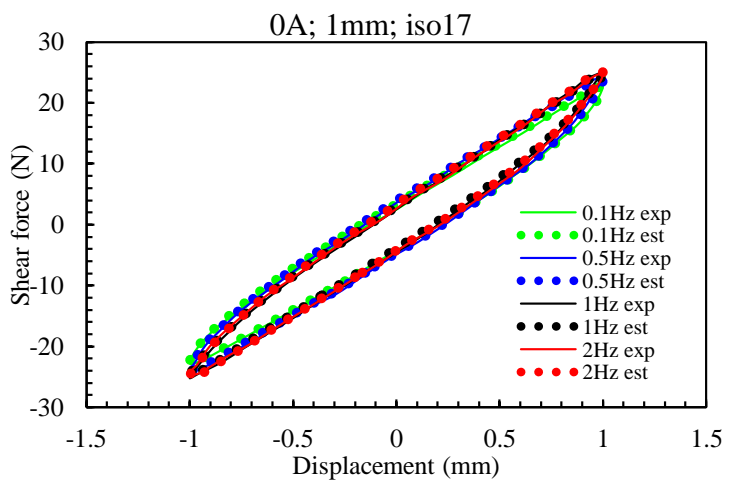

(a) Tests under different frequencies

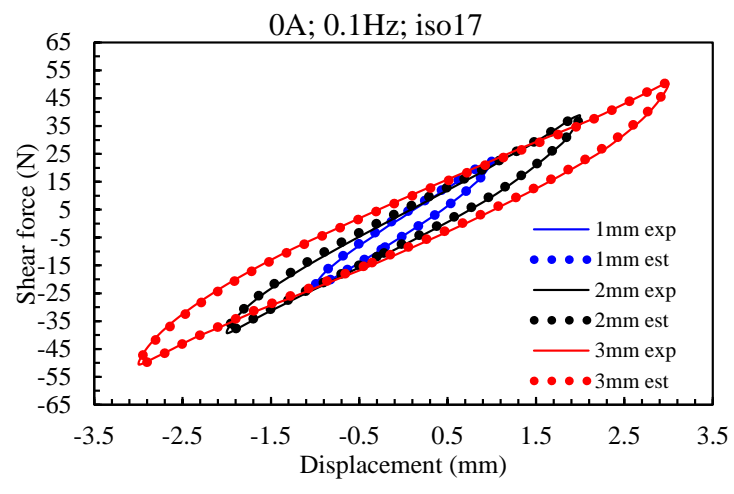

(b) Tests under different amplitudes

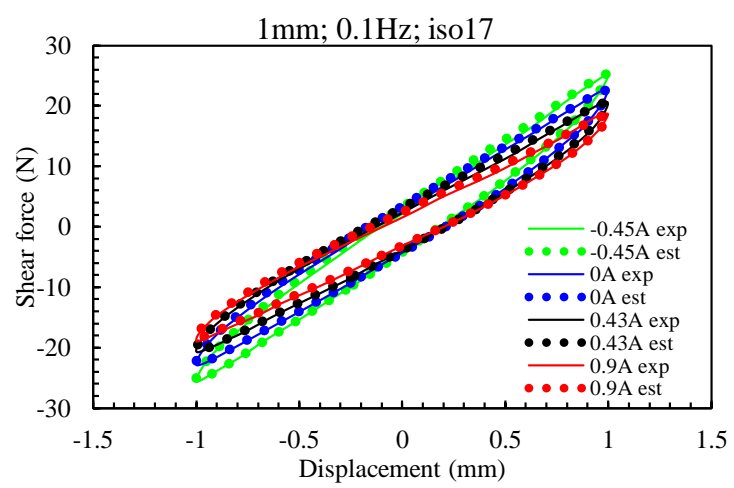

(c) Tests under different currents

Figure 12. Modelling results of iso 17 


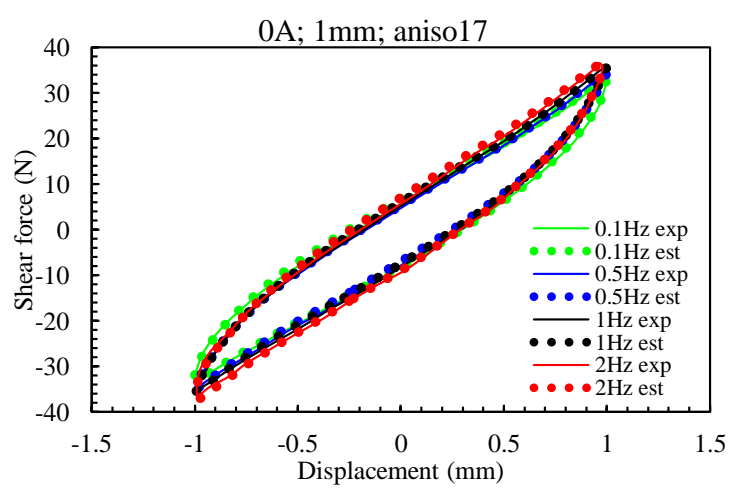

(a) Tests under different frequencies

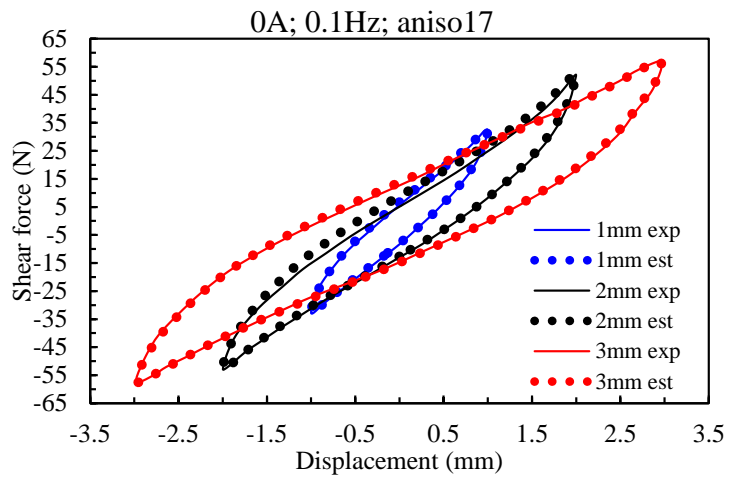

(b) Tests under different amplitudes

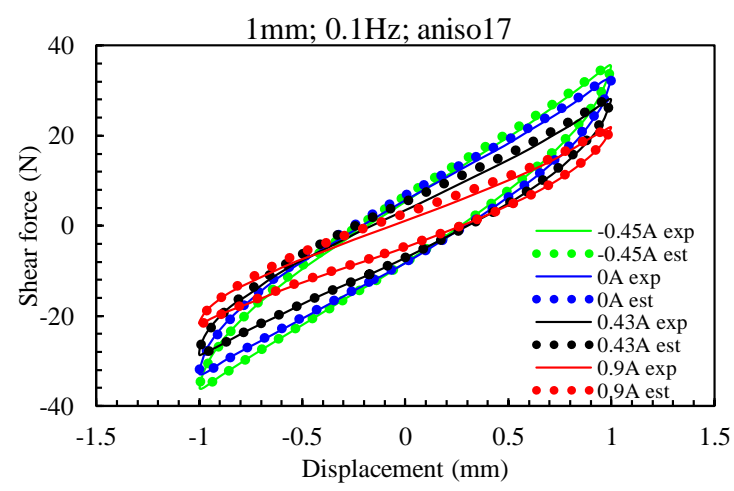

(c) Tests under different currents

Figure 13. Modelling results of aniso17

The identified model parameters were further analysed to reveal their dependencies on the applied magnetic field. In figure 14 and 15, results from the parameter identification process for the both iso17 and ansio17 samples are plotted to reveal the relationships between model parameters and the excitation frequency and the applied current. The parameters identified for isotropic and anisotropic showed identical and clear trends. For parameter $k$, as depicted in figure 14 (a) and figure 15 (a), with the value decrease with the increase of amplitude, but increase with the increase of excitation frequency. The reduce of applied current stands the intensifying of the magnetic field in the sample and increases the stiffness parameter. The damping parameter, $c$, values are summarised in figure 14 (b) and figure 15 (b). 

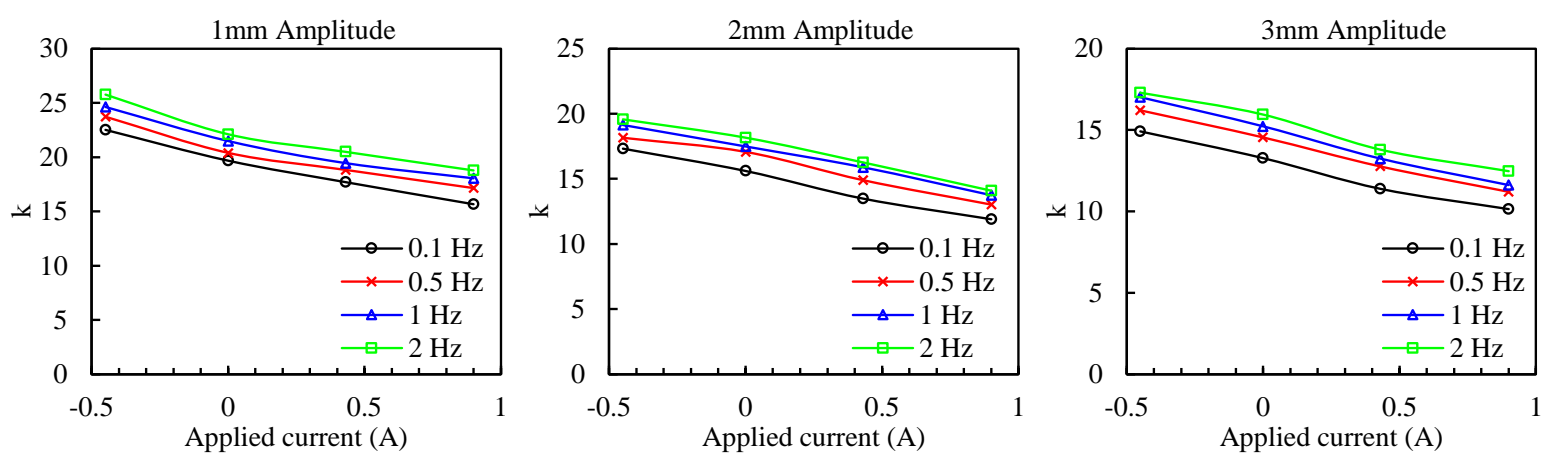

(a) Parameter k
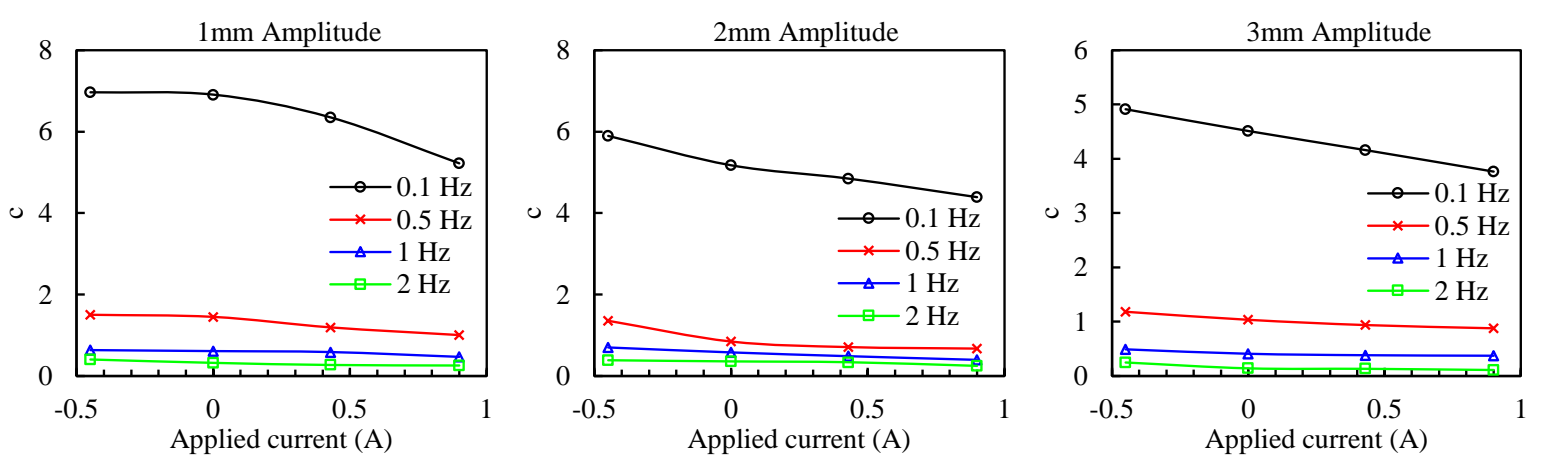

(b) Parameter c
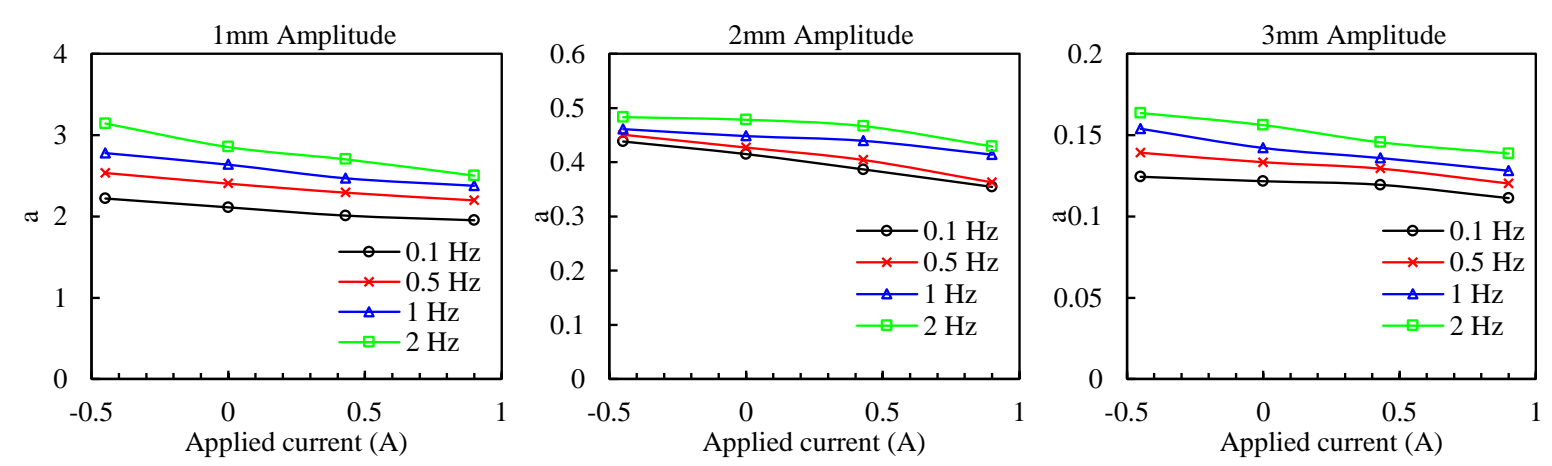

(c) Parameter a

Figure 14. Model parameters of the iso 17 sample 

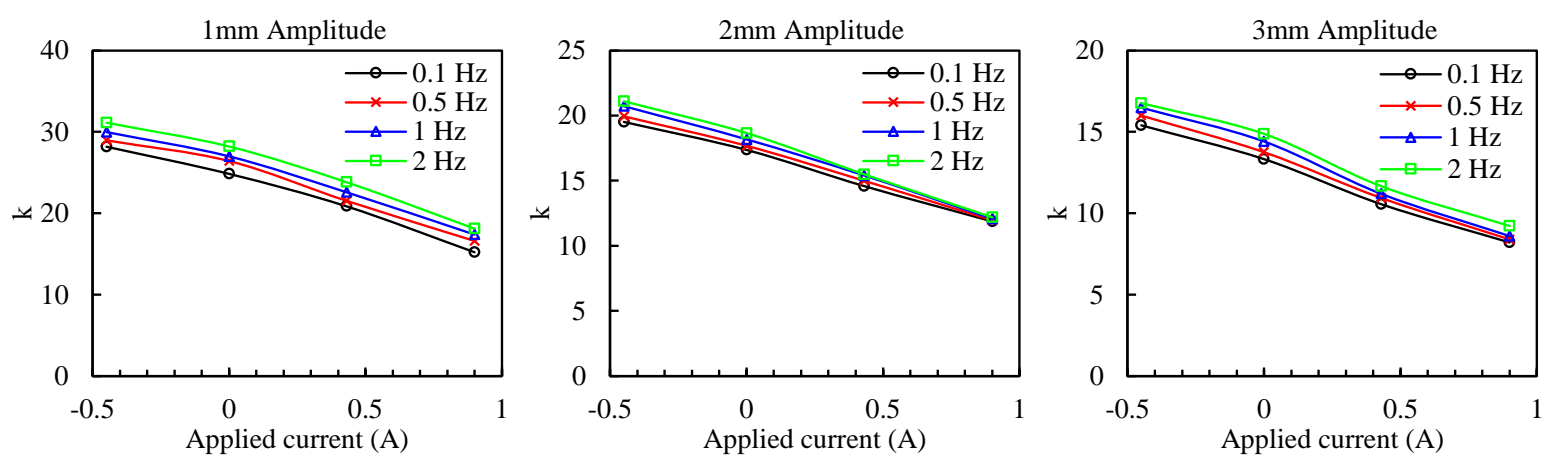

(a) Parameter $\mathrm{k}$
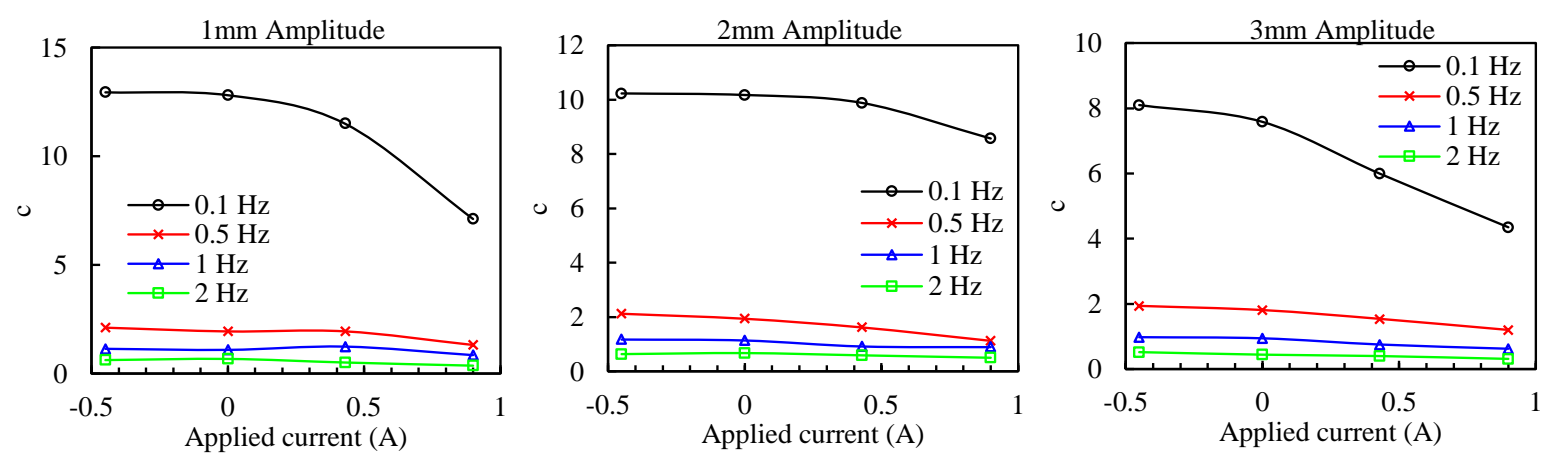

(b) Parameter c
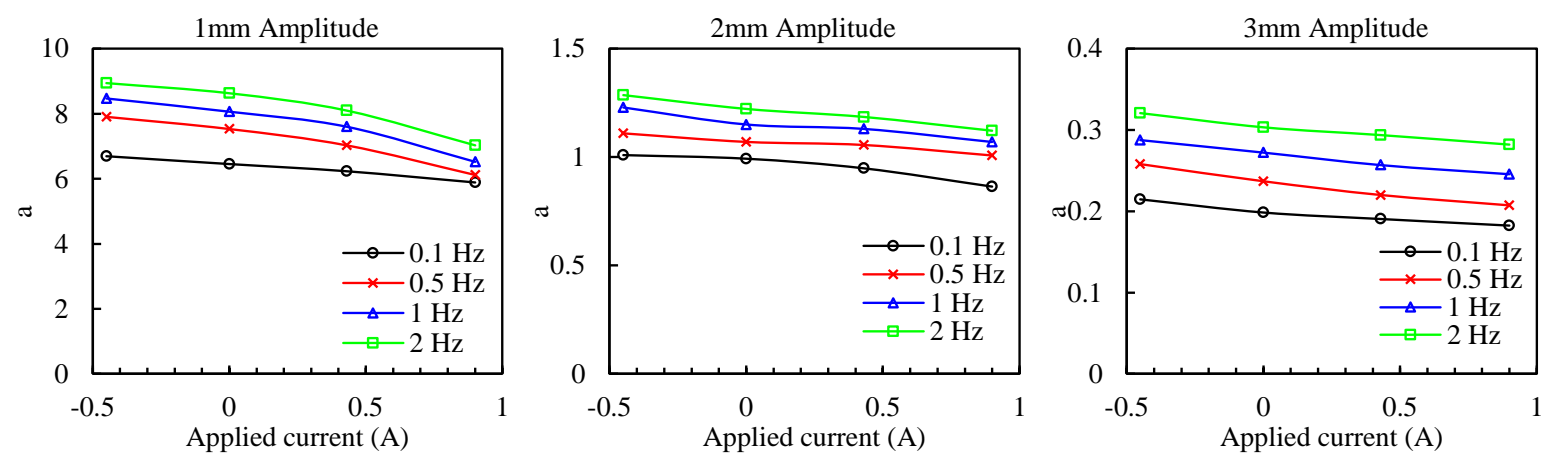

(c) Parameter a

Figure 15. Model parameters of the aniso17 sample

As the amplitude, excitation frequency and the applied current increase, the values of c decrease for both isotropic and anisotropic samples. These trends ideally agree with that of the calculated material properties, i.e., effective stiffness and equivalent damping, as discussed in section 4.3. As for the parameter $a$, which governs then magnitude of the strain stiffening effect, its value rises with the increase of excitation frequency and the intensifying of the magnetic field, however, decreases with the increase of excitation amplitude. For the isotropic sample, the 
smaller identified $a$ value than that of the anisotropic sample well reflects the lower strain stiffening effect of isotropic MREs.

\section{Conclusion}

This paper presented a novel shear test rig that features a hybrid magnets system. The system consists of a set of electromagnet coils and a PM. The implementation of PM provides a magnetic field without power supply. In this way, the maximum electric current required to provide the maximum adjustability of the MRE can be cut down and the overall energy consumption can be trimmed down. As the maximum applied current can be reduced, the risk of electric overload can be reduced; and, more compact design of the coil can also be realised. These advantages suggest that adopting the hybrid magnets system can greatly enhance the reliability, compatibility and running time of the magnetorheological-material-based adaptive devices. Based on the hybrid magnets concept, the designed test rig provides a magnetic field with $0.77 \mathrm{~T}$ flux density when no power supplied to the coils. When the coils are energized, the variation range of flux densities can be altered from $0.02 \mathrm{~T}$ to $1.01 \mathrm{~T}$. A series of MRE shear tests were performed by using this shear test rig. Six types of MRE were fabricated and tested, including isotropic and anisotropic samples with three different CIP volume fractions (17\%, $22 \%$ and $32 \%$ ). Results indicated that, when samples have the same CIP volume fraction, the anisotropic samples have higher stiffnesses, damping performances and better adjustability than that of the isotropic samples. Higher CIP content gives a higher effective stiffness and equivalent damping. The increase of excitation amplitude from $1 \mathrm{~mm}$ to $3 \mathrm{~mm}$ reduces the stiffness increase percentages from around $100 \%$ to $40 \%$ for anisotropic materials, and, $50 \%$ to $30 \%$ for isotropic materials. Moreover, the force-displacement hysteretic behaviours of both isotropic and anisotropic MRE can be well predicted by the strain stiffening phenomenological model effectively under the variances of excitation amplitude, frequency and applied current. 


\section{Reference}

[1] Li, Y., Li, J., Li, W., \& Samali, B. (2013). Development and characterization of a magnetorheological elastomer based adaptive seismic isolator. Smart Materials and Structures, 22(3), 035005.

[2] Li, Y., Li, J., Tian, T., \& Li, W. (2013). A highly adjustable magnetorheological elastomer base isolator for applications of real-time adaptive control. Smart Materials and Structures, 22(9), 095020.

[3] Deng, H. X., \& Gong, X. L. (2008). Application of magnetorheological elastomer to vibration absorber. Communications in nonlinear science and numerical simulation, 13(9), 1938-1947.

[4] Behrooz, M., Wang, X., \& Gordaninejad, F. (2014). Performance of a new magnetorheological elastomer isolation system. Smart Materials and Structures, 23(4), 045014.

[5] Gu, X., Yu, Y., Li, Y., Li, J., Askari, M., \& Samali, B. (2019). Experimental study of semi-active magnetorheological elastomer base isolation system using optimal neuro fuzzy logic control. Mechanical Systems and Signal Processing, 119, 380-398.

[6] Li, Y., Li, J., Li, W., \& Du, H. (2014). A state-of-the-art review on magnetorheological elastomer devices. Smart materials and structures, 23(12), 123001.

[7] Lu, X., Qiao, X., Watanabe, H., Gong, X., Yang, T., Li, W., ... \& Yin, Q. (2012). Mechanical and structural investigation of isotropic and anisotropic thermoplastic magnetorheological elastomer composites based on poly (styrene-b-ethylene-co-butyleneb-styrene)(SEBS). Rheologica acta, 51(1), 37-50.

[8] Sun, T. L., Gong, X. L., Jiang, W. Q., Li, J. F., Xu, Z. B., \& Li, W. H. (2008). Study on the damping properties of magnetorheological elastomers based on cis-polybutadiene rubber. Polymer Testing, 27(4), 520-526.

[9] Dargahi, A., Rakheja, S., \& Sedaghati, R. (2019). Development of a field dependent Prandtl-Ishlinskii model for magnetorheological elastomers. Materials \& Design, 166, 107608.

[10] Ju, B. X., Yu, M., Fu, J., Yang, Q., Liu, X. Q., \& Zheng, X. (2012). A novel porous magnetorheological elastomer: preparation and evaluation. Smart Materials and Structures, 21(3), 035001.

[11] Zhou, G. Y. (2003). Shear properties of a magnetorheological elastomer. Smart materials and structures, 12(1), 139. 
[12] Imaduddin, F., Li, Y., Mazlan, S. A., Sutrisno, J., Koga, T., Yahya, I., \& Choi, S. B. (2016). A new class of magnetorheological elastomers based on waste tire rubber and the characterization of their properties. Smart Materials and Structures, 25(11), 115002.

[13] Gong, X. L., Zhang, X. Z., \& Zhang, P. Q. (2005). Fabrication and characterization of isotropic magnetorheological elastomers. Polymer testing, 24(5), 669-676.

[14] Zając, P., Kaleta, J., Lewandowski, D., \& Gasperowicz, A. (2010). Isotropic magnetorheological elastomers with thermoplastic matrices: structure, damping properties and testing. Smart Materials and Structures, 19(4), 045014.

[15] Yang, J., Sun, S. S., Du, H., Li, W. H., Alici, G., \& Deng, H. X. (2014). A novel magnetorheological elastomer isolator with negative changing stiffness for vibration reduction. Smart materials and structures, 23(10), 105023.

[16] Sun, S., Deng, H., Yang, J., Li, W., Du, H., Alici, G., \& Nakano, M. (2015). An adaptive tuned vibration absorber based on multilayered MR elastomers. Smart materials and structures, 24(4), 045045.

[17] Li, S., Watterson, P., Li, Y., Wen, Q., \& Li, J. (2020). Improved magnetic circuit analysis of a laminated magnetorheological elastomer devices featuring both permanent magnets and electromagnets. Smart Materials and Structures.

[18] Li, W. H., Zhou, Y., \& Tian, T. F. (2010). Viscoelastic properties of MR elastomers under harmonic loading. Rheologica acta, 49(7), 733-740.

[19] Yu, Y., Li, Y., Li, J., \& Gu, X. (2016). A hysteresis model for dynamic behaviour of magnetorheological elastomer base isolator. Smart Materials and Structures, 25(5), 055029. 